\title{
Conselhos Municipais de Alimentação Escolar em Santa Catarina: caracterização e perfil de atuação
}

\author{
Municipal School Nutrition Councils in Santa Catarina, Brazil: \\ characterization and performance profile
}

Cristine Garcia Gabriel ${ }^{1}$

Manuella de Souza Machado ${ }^{2}$

Bethsáida de Abreu Soares Schmitz ${ }^{1}$

Arlete Catarina Tittoni Corso ${ }^{3}$

Gilberto Veras Caldeiras ${ }^{1}$

Francisco de Assis Guedes de Vasconcelos ${ }^{3}$

\footnotetext{
${ }^{1}$ Programa de PósGraduação em Saúde Pública, Centro de Ciências da Saúde, Universidade Federal de Santa Catarina. Campus Universitário, Trindade. 88040-970 Florianópolis SC. cris_ntr@hotmail.com ${ }^{2}$ Centro Colaborador em Alimentação e Nutrição do Escolar de Santa Catarina (CECANE/SC)

3 Departamento de Nutrição, Centro de Ciências da Saúde, Universidade Federal de Santa Catarina.
}

\begin{abstract}
The aim of this study is to assess Municipal School Nutrition Councils (CAEs) of the Brazilian School Nutrition Program (PNAE) in the state of Santa Catarina, Brazil. A cross-sectional study was conducted with a sample of municipalities in the state $(n=152 ; 52 \%)$. CAE presidents were interviewed and asked about the counselor profile, attributions performed and the relation of CAEs with other local councils. Poisson regression analysis was conducted to obtain unadjusted and adjusted prevalence ratios and the adjusted model adopted a hierarchic approach. The majority of councils analyzed their accounting reports, monitored resources and strived for quality of the CAEs at all levels. They coordinated the storage of food, participated in establishing the menu, monitored the purchasing of food and had an internal control system. However, only $22.4 \%$ carried out all their attributions once or more per year. A positive association was found between an adequate performance of the CAE and relations with other local councils. The awareness of the CAEs, as well as a stimulus to acting jointly with other councils and actors involved with food and nutrition security, are essential to ensure the right to healthy and adequate nutrition at school.
\end{abstract}

Key words Social control, School nutrition, $\mathrm{Mu}$ nicipal school nutrition council, Nutrition programs and policies
Resumo O objetivo deste estudo éavaliar os Conselhos Municipais de Alimentação Escolar (CAE) no Estado de Santa Catarina. Investigação transversal em amostra de municípios catarinenses ( $n$ $=152 ; 52 \%)$. Entrevistaram-se preferencialmente os presidentes dos CAE, pesquisando-se o perfil dos conselheiros, as atribuições realizadas e a relação do CAE com outras instâncias de controle social. Utilizou-se Regressão de Poisson para obter razões de prevalências bruta e ajustada. No modelo ajustado seguiu-se uma abordagem hierárquica. A maioria dos conselhos analisava a prestação de contas, fiscalizava os recursos, zelava pela qualidade do PNAE em todos os níveis, orientava o armazenamento dos alimentos, participava da elaboração do cardápio, acompanhava a compra de gêneros e tinha regimento interno. Entretanto, apenas $22,4 \%$ realizavam todas as atribuições com frequência maior ou igual à anual. Verificou-se associação positiva entre uma boa atuação do CAE e existência de relação com outros conselhos locais. A sensibilização dos CAE e o estímulo à atuação articulada com outros conselhos e atores envolvidos com a segurança alimentar e nutricional, são essenciais para a garantia do direito à alimentação saudável e adequada nas escolas.

Palavras-chave Controle social, Alimentação escolar, Conselho de alimentação escolar, Programas e políticas de nutrição e alimentação 


\section{Introdução}

Em resposta à mobilização social, a Constituição Federal Brasileira de 1988 estabeleceu preceitos que possibilitaram a descentralização da gestão das políticas públicas e a participação da sociedade nos processos de tomada de decisão ${ }^{1}$. O controle social da gestão pública busca firmar-se como um espaço de cogestão entre Estado e sociedade, propiciando o exercício da cidadania ativa ${ }^{2,3}$.

Experiências de democracia participativa vivenciadas por distintas modalidades de conselhos municipais indicam teoricamente um salto na instituição democrática. Todavia, na prática, estas têm ainda um longo caminho a percorrer antes de consolidarem-se como realidade efetiva ${ }^{3,4}$.

Toma-se como estudo o Conselho de Alimentação Escolar (CAE), órgão colegiado de caráter fiscalizador, permanente, deliberativo e de assessoramento, a ser instituído nos estados, no Distrito Federal e nos municípios, visando à efetivação do Programa Nacional de Alimentação Escolar (PNAE) $)^{5}$.

O PNAE destaca-se como um dos maiores programas mundiais na área de alimentação escolar. Teve sua execução descentralizada a partir de 1994, condicionada à criação dos $\mathrm{CAE}^{6-9}$. A descentralização do Programa possibilitou a ampliação do exercício dos direitos e maior autonomia da gestão municipal, com maior controle dos recursos públicos ${ }^{10,11}$.

O PNAE objetiva contribuir para o crescimento e o desenvolvimento biopsicossocial, a aprendizagem, o rendimento escolar e a formação de hábitos alimentares saudáveis dos alunos de toda a educação básica pública, por meio de ações de educação alimentar e nutricional e da oferta de refeições ${ }^{12}$.

A responsabilidade de fiscalização do PNAE cabe ao seu órgão gestor, o Fundo Nacional de Desenvolvimento da Educação (FNDE), ao Tribunal de Contas da União, à Controladoria-Geral da União, ao Ministério Público e à sociedade, por meio do $\mathrm{CAE}^{5}$.

Entretanto, a possibilidade do CAE refletir a visão da comunidade só se efetivará caso os membros se constituam a partir da mobilização e discussão social ${ }^{8}$. Neste sentido, a proposta de composição do CAE contida na Resolução no 38/2009/ FNDE avança no sentido de propor a articulação de representantes de diferentes segmentos (poder executivo, profissionais da educação e discentes, representação de pais de alunos e da sociedade civil $)^{5}$.

Os estudos no país sobre a atuação dos CAE são escassos ${ }^{8,9,13}$, sendo este o primeiro em Santa
Catarina. O propósito deste artigo foi avaliar os Conselhos Municipais de Alimentação Escolar no Estado de Santa Catarina, procurando identificar e analisar suas características e perfil de atuação.

\section{Métodos}

Esta investigação possui delineamento transversal, realizada de maio a julho de 2008 .

Calculou-se amostra representativa dos 293 municípios de Santa Catarina, utilizando-se como critério o porte dos municípios, classificando-os em: municípios de pequeno porte (menos de 20 mil habitantes), médio porte (20 até 100 mil habitantes) e, grande porte (mais de 100 mil habitantes).

Com base nessa classificação, Santa Catarina possuía somente 10 municípios de grande porte, definindo-se que esses municípios comporiam a amostra, independente dos cálculos. Realizou-se o cálculo amostral para os estratos de municípios de pequeno e médio porte. A amostra foi definida como: 10 municípios de grande porte, 23 de médio porte e 120 de pequeno porte, totalizando 153 municípios.

A coleta das informações ocorreu por meio de entrevista estruturada, preferencialmente junto ao presidente do CAE, sendo entrevistado outro conselheiro, na impossibilidade deste.

A equipe responsável pela coleta foi composta por quatro nutricionistas treinados, sendo realizado teste piloto em três municípios, não participantes da amostra final.

O questionário constituiu-se de questões fechadas e exploratórias. Para a caracterização do perfil dos membros do CAE utilizaram-se as variáveis: sexo, faixa etária, grau de escolaridade, renda mensal estratificada em salários mínimos, profissão, representação e cargo ocupado no CAE, experiência prévia em CAE ou outra instância de controle social. Além destas variáveis, caracterizaram-se os conselhos segundo a existência de vínculo do presidente/vice-presidente com os poderes executivo/legislativo, a participação prévia em capacitações relativas à atuação do CAE, a existência de regimento interno, a frequência e local das reuniões e a interação com outros conselhos locais.

Avaliaram-se as atribuições desempenhadas pelo CAE com base na legislação vigente na época do estudo (Resolução no $32 / 2006 /$ FNDE) ${ }^{14}$. Assim, investigou-se a fiscalização dos recursos; o acompanhamento e o monitoramento da aquisição dos produtos; o zelo pela qualidade dos 
produtos em todos os níveis; a orientação sobre o armazenamento dos gêneros; a divulgação em locais públicos dos recursos financeiros e o recebimento e a análise da prestação de contas ${ }^{14}$. Foi questionada também a frequência de realização destas ações, além do conhecimento do conselheiro sobre a atuação do nutricionista como responsável técnico pelo PNAE e a participação do CAE na elaboração do cardápio.

Realizou-se estatística descritiva das variáveis de caracterização dos conselheiros e cumprimento das atribuições do CAE. Para o desfecho foi considerado como conselho adequado aquele que realizava as atividades previstas na legislação em vigor $^{14}$, com exceção do item relativo à divulgação de atividades, sendo essas ações executadas com periodicidade maior do que uma vez ao ano, com a intenção de não considerar atividades pontuais e/ou aleatórias. Foram obtidas as Razões de Prevalências (RP) bruta e ajustada, e seus respectivos intervalos de confiança de $95 \%$, por meio da Regressão de Poisson. No modelo ajustado seguiu-se uma abordagem hierárquica, sendo o ajuste realizado pelo Produto Interno Bruto (PIB) e porte populacional do município (Figura 1). Esta análise permite ajuste por fatores de confusão. Incluíram-se todas as variáveis no modelo, por julgar que todas poderiam influenciar no desfecho, excluindo-se, posteriormente, aquelas que apresentavam valor $p<0,20$. Os dados obtidos foram digitados e analisados nos programas Epi-Data versão 3.1 e Stata 11.0.

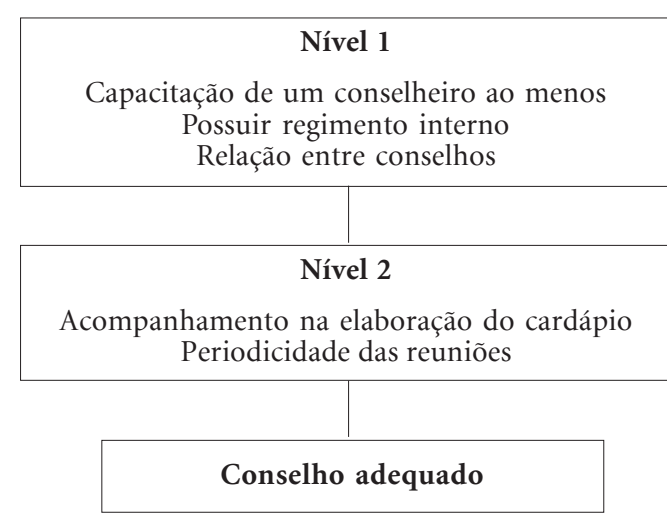

Figura 1. Modelo hierárquico das variáveis usadas para categorização do desfecho (conselho adequado) do estudo sobre os Conselhos de Alimentação Escolar em Santa Catarina.
A pesquisa foi aprovada pelo Comitê de Ética em Pesquisa com Seres Humanos da Universidade Federal de Santa Catarina, de acordo com a Resolução 196/96 do Conselho Nacional de Saúde ${ }^{15}$.

\section{Resultados}

\section{Características gerais}

Realizou-se a coleta em 152 (99,3\%) municípios, sendo 121 de pequeno porte $(79,6 \%), 21$ de médio porte $(13,8 \%)$ e 10 de grande porte $(6,6 \%)$. A pequena diferença entre o cálculo amostrado deve-se à exclusão de um município e à substituição de dois de médio porte devido à dificuldade de contato, após repetidas tentativas.

A Tabela 1 traça um perfil geral dos conselheiros. A maioria pertencia ao sexo feminino (80,9\%); com nível superior completo (74,3\%); eram presidentes ou vice-presidentes do CAE $(61,2 \%)$, representantes de professores (50,7\%); com renda mensal entre 3 e 5 salários mínimos (SM) $(40,8 \%)$ e, com experiência prévia em conselhos (inclusive o CAE) (59,2\%). Possuíam idade média de 38,1 anos.

Além disso, 18,4\% $(\mathrm{n}=28)$ dos conselheiros afirmaram que o presidente do CAE possuía vínculo com o poder executivo ou legislativo; $47,5 \%$ $(\mathrm{n}=68)$ afirmaram que pelo menos algum conselheiro participara de capacitação na área; e 68,3\% ( $\mathrm{n}=97)$ afirmaram manter relação com outros conselhos locais (dados não mostrados em tabelas).

\section{Atribuições do CAE}

A Tabela 2 mostra atribuições dos CAE, destacando-se a análise da prestação de contas $(95,4 \%)$, seguida da fiscalização dos recursos federais $(91,4 \%)$. Quanto à periodicidade da fiscalização, mais da metade dos conselhos $(54,7 \%)$ fiscalizavam os recursos com periodicidade igual ou superior a seis meses. A maioria referiu que o CAE zelava pela qualidade dos produtos em todos os níveis $(84,9 \%)$ e orientava sobre armazenamento dos alimentos $(66,4 \%)$. As frequências de tais atividades mostraram-se similares, oscilando entre mensais e semestrais. O acompanhamento da aquisição dos alimentos para o PNAE foi a atribuição executada com menor frequência, mensal ou inferior $(41,1 \%)$, mas ainda realizada pela maioria dos conselhos $(59,2 \%)$. Dentre os conselheiros, $27 \%$ referiram que o CAE divulgava em locais públicos os recursos do Pro- 
grama, enquanto praticamente metade dos conselhos reunia-se bimestralmente e quadrimestralmente $(49,4 \%)$.

A maioria $(n=125 ; 82,2 \%)$ referiu conhecer o nutricionista responsável pela alimentação es-

Tabela 1. Caracterização dos conselheiros segundo variáveis socioeconômicas e função no Conselho de Alimentação Escolar (CAE). Santa Catarina, 2008.

\begin{tabular}{|c|c|c|}
\hline Variáveis & $\mathbf{n}$ & $\%$ \\
\hline \multicolumn{3}{|l|}{ Sexo } \\
\hline Feminino & 123 & 80,9 \\
\hline Masculino & 29 & 19,1 \\
\hline \multicolumn{3}{|l|}{ Faixa Etária } \\
\hline Sem Informação & 3 & 1,9 \\
\hline Até 30 anos & 21 & 13,6 \\
\hline De 31 a 50 anos & 113 & 73,4 \\
\hline Acima de 50 anos & 15 & 9,7 \\
\hline \multicolumn{3}{|l|}{ Escolaridade } \\
\hline Não estudou & 1 & 0,7 \\
\hline Ensino fundamental incompleto & 5 & 3,3 \\
\hline Ensino fundamental completo & 4 & 2,6 \\
\hline Ensino médio incompleto & 2 & 1,3 \\
\hline Ensino médio completo & 13 & 8,6 \\
\hline Superior incompleto & 14 & 9,2 \\
\hline Superior completo & 113 & 74,3 \\
\hline \multicolumn{3}{|l|}{ Faixa de renda mensal } \\
\hline Não informou & 5 & 3,3 \\
\hline Até 1 salário mínimo (SM) & 4 & 2,6 \\
\hline Entre 1 e $2 \mathrm{SM}$ & 21 & 13,8 \\
\hline Entre 2 e $3 \mathrm{SM}$ & 32 & 21,0 \\
\hline Entre 3 e $5 \mathrm{SM}$ & 62 & 40,8 \\
\hline Entre 5 e $7,5 \mathrm{SM}$ & 20 & 13,2 \\
\hline Acima de $7,5 \mathrm{SM}$ & 7 & 4,6 \\
\hline Não tem renda própria & 1 & 0,7 \\
\hline \multicolumn{3}{|l|}{ Representação no CAE } \\
\hline Sem informação & 1 & 0,7 \\
\hline Poder executivo & 26 & 17,1 \\
\hline Poder legislativo & 2 & 1,3 \\
\hline Pais de alunos & 31 & 20,4 \\
\hline Professores & 77 & 50,7 \\
\hline Outros segmentos da sociedade civil & 15 & 9,9 \\
\hline \multicolumn{3}{|l|}{ Cargo no CAE } \\
\hline Presidente & 82 & 54,0 \\
\hline Vice-presidente & 11 & 7,2 \\
\hline Membro & 59 & 38,8 \\
\hline \multicolumn{3}{|l|}{$\begin{array}{l}\text { Experiência prévia em conselhos de } \\
\text { controle social }\end{array}$} \\
\hline $\operatorname{Sim}$ & 90 & 59,2 \\
\hline Não & 60 & 39,5 \\
\hline Não Soube Informar & 2 & 1,3 \\
\hline \multicolumn{3}{|l|}{ Experiência prévia no CAE } \\
\hline Sim & 56 & 36,4 \\
\hline Não & 94 & 61,0 \\
\hline Não Soube Informar & 2 & 1,3 \\
\hline Total & 152 & 100,0 \\
\hline
\end{tabular}

colar no município. Dentre esses, 80\% $(\mathrm{n}=100)$ referiram ter bom contato com o profissional (dados não apresentados).

Ainda, 96,1\% ( $\mathrm{n}=146)$ dos conselheiros mencionaram o nutricionista como o responsável técnico pela elaboração dos cardápios, sendo que $62,4 \%(n=93)$ referiram participação do CAE nesta etapa. Verificou-se que $96,7 \%(n=147)$ dos conselheiros relataram que os cardápios respeitavam os hábitos regionais dos escolares (dados não apresentados).

Dos entrevistados, 53,6\% $(\mathrm{n}=80)$ referiram que o CAE possuía regimento interno, e 22,4\% $(\mathrm{n}=34)$ não sabiam se o regimento existia. Quanto ao regimento, $85 \%(\mathrm{n}=68)$ relataram que o mesmo foi aprovado em assembleia; 27,5\% (n = 22) referiram alguma atualização deste durante o seu mandato e $85 \%(n=68)$ informaram que o regimento é utilizado pelo CAE. Observou-se que $42,5 \%(\mathrm{n}=34)$ afirmaram já ter lido o regimento, recordando-se dele, versus $46,3 \%(\mathrm{n}=37)$ que relataram ter lido há algum tempo, não recordando e, $11,2 \%(n=9)$ que nunca o leram.

Verificou-se que $22,4 \%$ dos CAE foram considerados como conselho adequado, uma vez que realizavam todas as atribuições previstas na legislação em vigor na época do estudo ${ }^{14}$, com frequência maior de uma vez ao ano, (exceto a atividade de divulgação em locais públicos dos recursos gastos) (dados não apresentados em tabelas).

A Tabela 3 mostra os fatores associados a um conselho adequado, conforme modelo hierárquico da Figura 1. Verificou-se aumento significativo de chances de melhor atuação do conselho $(3,2 ; 95 \%$; IC:1,1-8,6) apenas para a variável "existência de relação do CAE com demais conselhos locais". A ação de "acompanhar a elaboração dos cardápios” esteve positivamente associada à boa atuação do CAE na análise bruta (2,2;95\%; IC:1,0-4,8), perdendo significância na ajustada.

\section{Discussão}

Apesar de não estar oficialmente definido o número de reuniões que o conselho deva realizar durante o $\mathrm{ano}^{5,14}$, sugerem-se reuniões mensais ou várias vezes ao ano ${ }^{13}$. Pipitone et al. $^{8}$ verificaram frequência mensal (39\%), superior à encontrada em Santa Catarina (26\%). No presente estudo, 49,4\% dos CAE reuniam-se com frequência de dois a quatro meses $(49,4 \%)$. Na Bahia, $14 \%$ dos municípios referiram que o CAE nunca se reuniu após sua constituição9 ${ }^{9}$ A pesquisa sobre o perfil 
Tabela 2. Atribuições realizadas pelos Conselhos de Alimentação Escolar (CAE). Santa Catarina, 2008.

\begin{tabular}{|c|c|c|c|c|c|c|}
\hline \multirow[b]{2}{*}{ Atribuições do CAE } & \multirow[b]{2}{*}{$\begin{array}{l}\text { Total que } \\
\text { cumprem } \\
\text { n }(\%)\end{array}$} & \multicolumn{4}{|c|}{ Periodicidade } & \multirow[b]{2}{*}{$\begin{array}{l}\text { Não } \\
\text { sabem } \\
\text { n } \quad(\%)\end{array}$} \\
\hline & & $\begin{array}{l}\text { Mensal ou } \\
\text { inferior } \\
\text { n }(\%)\end{array}$ & $\begin{array}{l}\text { Bimestral à } \\
\text { quadrimestral } \\
\text { n }(\%)\end{array}$ & $\begin{array}{l}\text { Semestral } \\
\text { ou mais } \\
\text { n }(\%)\end{array}$ & $\begin{array}{c}\text { Cumprem atribuição } \\
\text { apenas na escola onde } \\
\text { atuam, sem cronograma } \\
\text { n }(\%)\end{array}$ & \\
\hline $\begin{array}{l}\text { Fiscaliza a aplicação dos } \\
\text { recursos }\end{array}$ & $139(91,4)$ & $21 \quad(15,1)$ & $35(25,2)$ & $76(54,7)$ & - & $7(5,0)$ \\
\hline $\begin{array}{l}\text { Acompanha e monitora a } \\
\text { compra dos alimentos }\end{array}$ & $90(59,2)$ & $37(41,1)$ & $19(21,2)$ & $26(28,8)$ & - & $8(8,9)$ \\
\hline $\begin{array}{l}\text { Zela pela qualidade dos } \\
\text { produtos, em todos os níveis }\end{array}$ & $129(84,9)$ & $46(35,7)$ & $32(24,8)$ & $37(28,7)$ & $13(10,1)$ & $1(0,8)$ \\
\hline $\begin{array}{l}\text { Orienta sobre o } \\
\text { armazenamento dos gêneros } \\
\text { alimentícios }\end{array}$ & $101(66,4)$ & $34(33,7)$ & $27(26,8)$ & $37(36,6)$ & $3(3,0)$ & - \\
\hline $\begin{array}{l}\text { Divulga em locais públicos os } \\
\text { recursos financeiros }\end{array}$ & $41(27,0)$ & - & - & - & & - \\
\hline $\begin{array}{l}\text { Recebe e analisa a prestação } \\
\text { de contas }\end{array}$ & $145(95,4)$ & - & - & - & & - \\
\hline Reuniões* $^{*}$ & $152(100)$ & $40(26,3)$ & $75(49,4 \%)$ & $37(24,3)$ & - & - \\
\hline
\end{tabular}

* O item reuniões não consta no artigo 17 como atribuição do CAE, mas é citado no artigo 18, referente ao regimento interno (Resolução n³2/ 2006/FNDE) $)^{12}$

Tabela 3. Fatores associados a um conselho de alimentação escolar adequado. Razões de prevalência brutas e ajustadas (RP, IC 95\%). Santa Catarina, 2008.

\begin{tabular}{|c|c|c|c|c|}
\hline \multirow{2}{*}{ Variável } & \multicolumn{2}{|c|}{ Análise bruta } & \multicolumn{2}{|c|}{ Análise ajustada } \\
\hline & RP (IC 95\%) & $\mathbf{p}^{*}$ & RP (IC 95\%) & $\mathbf{p}^{*}$ \\
\hline Capacitação de pelo menos 1 conselheiro & & 0,754 & & $0,914^{* *}$ \\
\hline Não & 1,0 & & 1,0 & \\
\hline $\operatorname{Sim}$ & $1,1(0,6-2,0)$ & & $1,0(0,5-1,8)$ & \\
\hline Conhecimento sobre regimento interno & & 0,258 & & $0,807^{* *}$ \\
\hline $\operatorname{Sim}$ & 1,0 & & 1,0 & \\
\hline Não & $0,9(0,5-1,9)$ & & $1,2(0,6-2,5)$ & \\
\hline Não sabe & $0,6(0,2-1,4)$ & & $0,8(0,3-2,0)$ & \\
\hline Relação com conselhos locais & & 0,016 & & $0,027^{* *}$ \\
\hline Não & 1,0 & & 1,0 & \\
\hline Sim & $3,4(1,3-9,0)$ & & $3,2(1,1-8,6)$ & \\
\hline Acompanha a elaboração do cardápio & & 0,040 & & $0,168^{* * *}$ \\
\hline Não & 1,0 & & 1,0 & \\
\hline Sim & $2,2(1,0-4,8)$ & & $1,8(0,8-4,2)$ & \\
\hline Periodicidade das reuniões & & 0,111 & & $0,714^{* * *}$ \\
\hline Ao menos mensal & 1,0 & & 1,0 & \\
\hline Bimestral ou quadrimestral & $1,1(0,6-2,1)$ & & $1,1(0,6-2,2)$ & \\
\hline Semestral ou mais & $0,4(0,1-1,3)$ & & $0,7(0,2-2,3)$ & \\
\hline
\end{tabular}

da gestão pública do PNAE das 670 prefeituras inscritas no Prêmio Gestor Eficiente da Alimentação Escolar considerou a atuação dos CAE razoável, sendo o número médio de reuniões de sete (em 2004) e seis (em 2005) ${ }^{13}$. Ressalta-se a relevância dos poucos dados comparativos disponíveis, visto a necessidade de encontros regulares do CAE para o devido acompanhamento do PNAE. Caso contrário, corre-se o risco de reuniões apenas de cunho anual, obrigatórias pela legislação, para averiguação da prestação de contas.

Ainda, a pesquisa com as prefeituras destaca casos onde a participação dos conselheiros foi determinante para redirecionar a gestão pública, 
evitando a terceirização do PNAE e exigindo contratação de nutricionista. Nesses casos os conselhos chegaram a reunir-se mais de 20 vezes ao ano ${ }^{13}$.

Pipitone et al. verificaram como principais atribuições dos conselhos a fiscalização da aplicação dos recursos (77\%), o acompanhamento do Programa nas escolas (63\%) e a colaboração nos cardápios $(50 \%)^{8}$. Estudo na Bahia ${ }^{9}$ indicou como principal atribuição a fiscalização dos recursos e o acompanhamento da distribuição dos gêneros $(17 \%)$, relatando que $15 \%$ dos conselhos não desempenhavam atividade alguma, estando constituídos oficialmente, mas sem atuação efetiva ${ }^{9}$.

Em Santa Catarina, a principal atribuição foi a análise da prestação de contas (95\%), seguida da fiscalização dos recursos (91\%), reafirmando indícios de que a fiscalização financeira é a principal atribuição executada. Verifica-se a necessidade de formações dos conselheiros, para uma devida apropriação das outras atribuições do CAE, e da relevância da realização dessas para o desenvolvimento satisfatório do Programa.

Um alto percentual ( $85 \%$ ) de conselheiros em Santa Catarina referiu que o CAE zela pela qualidade dos produtos, principalmente por meio das visitas às escolas. Destes, um pequeno percentual (10\%), referiu acompanhar o Programa somente nas escolas aonde trabalham.

Em Santa Catarina observou-se 22,4\% de CAE classificados como conselho adequado. Embora a maioria dos CAE realizasse as cinco atribuições consideradas na legislação ${ }^{14}$, pelo critério de análise do estudo, a frequência de execução dessas estava aquém do entendido como mínimo para uma atuação efetiva.

$\mathrm{O}$ adequado planejamento e acompanhamento da execução dos cardápios são fatores importantes para o alcance dos objetivos do Programa, sendo o cardápio um instrumento para a formação de hábitos alimentares ${ }^{16-18}$. Na Bahia, em 59\% dos municípios os cardápios eram os mesmos para todas as escolas, independentemente se urbanas ou rurais ${ }^{9}$.

Chaves et al. ${ }^{19}$ analisando a utilização de preparações regionais nos cardápios, destacaram que $86 \%$ dos cardápios da região Sul e 38\% da região Norte contemplavam pelo menos uma preparação regional semanal. A frequência de preparações regionais nos cardápios indica o respeito à cultura alimentar, preconizado pelo PNAE ${ }^{5,12,20}$.

No presente estudo, apesar da maioria dos conselheiros reconhecerem a responsabilidade técnica do nutricionista na elaboração dos car- dápios, e considerarem que os cardápios respeitam os hábitos regionais, apenas $61 \%$ referiram que o CAE participa desta etapa. São necessários estímulos e orientações para os CAE acompanharem a elaboração dos cardápios, possibilitando observação do respeito aos hábitos alimentares, à vocação agrícola regional e a utilização preferencial de produtos in natura ${ }^{8}$.

Mais da metade dos entrevistados (59\%) referiram experiência prévia em algum conselho de cunho social, sugerindo relação com o fato de $68 \%$ dos conselheiros afirmarem que o CAE mantinha relação com outros conselhos locais. Entende-se que a experiência prévia em conselhos possa influenciar positivamente o CAE, possivelmente em função do acúmulo de experiência no campo das políticas públicas. No entanto, tal associação necessita de maior investigação e reflexão.

No modelo hierárquico de análise construído, verificou-se que a existência de relação do CAE com os demais conselhos locais aumentou significativamente as chances de atuação satisfatória. O Artigo 27 da Resolução no 38/2009/FNDE refere, dentre as atribuições do CAE, que estes poderão desenvolver suas atribuições em regime de cooperação com os Conselhos de Segurança Alimentar e Nutricional (CONSEA) e demais conselhos afins ${ }^{5}$. Os conselhos de controle social são instâncias que objetivam aumentar a transparência e a efetividade das políticas públicas. Assim, entende-se que atuações conjuntas poderiam fortalecer estes espaços por meio da troca de saberes e experiências. As demais variáveis que compuseram o modelo final de análise do presente estudo não mantiveram associação no ajuste final, reforçando a relevância e a necessidade de estudos que possam aprofundar tais achados.

Uma das questões que pode comprometer a atuação dos conselhos sociais é o pouco conhecimento destes pela população, com baixa divulgação das atividades realizadas. Isto leva a um desconhecimento sobre os objetivos, as funções e a atuação do conselho, representando, ao final, uma baixa adesão da população nos processos decisórios locais ${ }^{21}$.

Este estudo não investigou o processo de seleção dos membros do CAE, sendo este um ponto importante a ser observado em estudos futuros. Os depoimentos encontrados no estudo da Bahia ${ }^{9}$ sugerem limitada transparência e forte presença do executivo municipal na escolha dos representantes, o que é típico de locais aonde o controle social ainda não é tão atuante. Em Santa Catarina observou-se que $18,4 \%$ dos CAE possuíam presidentes com vínculo com o poder exe- 
cutivo ou legislativo, sendo isto irregular conforme legislação da época ${ }^{14}$. Ressalta-se que a legislação atual não mais inclui o legislativo como uma das representações do conselho ${ }^{5}$. Martins et al. $^{21}$ salientam que um dos problemas mais frequentes e difíceis de serem solucionados é o relacionado à composição, representação e representatividade dos conselhos.

Pipitone et al. $^{8}$ reforça que o conjunto dos resultados encontrados no país evidencia a necessidade de uma atuação mais efetiva dos CAE. A pesquisa sobre o perfil da gestão pública das prefeituras inscritas no Prêmio Gestor Eficiente da Alimentação Escolar mostrou que um fator determinante e diferenciador nas administrações vencedoras foi a participação social, seja por meio da atuação ativa do CAE ou pelo envolvimento de pais de alunos, professores, merendeiros e agricultores locais ${ }^{13}$.

\section{Conclusão}

Em Santa Catarina, verificaram-se pontos satisfatórios acerca da atuação dos CAE, sendo que algumas questões importantes ainda necessitam de aperfeiçoamento. Dentre estas destacam-se o desconhecimento sobre o conteúdo e a impor- tância do Regimento Interno; a não divulgação sobre os recursos gastos com o Programa; a baixa participação na elaboração do cardápio; bem como uma maior periodicidade na fiscalização dos recursos federais, não devendo ser esta atividade restrita apenas ao período da análise da prestação de contas.

Espera-se que este estudo propicie subsídios para o aperfeiçoamento das ações de cunho oficial para as instâncias do governo federal (FNDE), no que concerne a incentivos e capacitações, assim como para os gestores dos municípios do estado de Santa Catarina, contribuindo para a melhoria da gestão e do controle social relativo à execução do PNAE.

Reforça-se a importância da relação de parceria encontrada neste estudo entre o CAE e os demais conselhos de controle social, que possuem como objetivo comum aprimorar as políticas públicas de acordo com as demandas sociais locais. Além disso, destaca-se a necessidade de atuação interligada de nutricionistas e conselheiros, os quais, em conjunto com os profissionais da educação e saúde e demais membros da comunidade escolar, se constituem em agentes fundamentais no estímulo aos alunos à prática de hábitos alimentares saudáveis, com vistas à garantia da segurança alimentar e nutricional.

\section{Colaboradores}

CG Gabriel e MS Machado participaram da concepção e desenho do projeto, coleta, análise e interpretação dos dados, redação do artigo. BAS Schmitz, FAG Vasconcelos, ACT Corso e GV Caldeiras participaram da concepção e desenho do projeto e, revisão crítica do texto.

\section{Agradecimentos}

Ao Fundo Nacional de Desenvolvimento da Educação por ter financiado o projeto que originou este artigo. 


\section{Referências}

1. Van Stralen CJ, Lima AMD, Sobrinho DF, Saraiva LES, Van Stralen TBS, Belisário SA. Conselhos de Saúde: efetividade do controle social em municípios de Goiás e Mato Grosso do Sul. Cien Saude Colet 2006; 11(3):621-632.

2. Silva AX, Cruz EA, Melo V. A importância estratégica da informação em saúde para o exercício do controle social. Cien Saude Colet 2007; 12(3):683-688.

3. Cotta RMM, Cazal MM, Rodrigues JFC. Participação, Controle Social e Exercício da Cidadania: a (des)informação como obstáculo à atuação dos conselheiros de saúde. Physis 2009; 19(2):419-438.

4. Kleba MEd, Matielo A, Comerlatto D, Renk E, Colliselli L. O papel dos conselhos gestores de políticas públicas: um debate a partir das práticas em Conselhos Municipais de Chapecó (SC). Cien Saude Colet 2010; 15(3):793-802.

5. Brasil. Fundo Nacional de Desenvolvimento da Educação (FNDE). Resolução/CD/FNDE no 38, de 16 de julho de 2009. Dispõe sobre o atendimento da alimentação escolar aos alunos da educação básica no Programa Nacional de Alimentação Escolar - PNAE. [acessado 2011 nov 15]. Disponível em: ftp://ftp.fnde.gov.br/web/resolucoes_2009/ res038_16072009.pdf.

6. Brasil. Ministério da Educação (ME). Fundo Nacional de Desenvolvimento da Educação (FNDE). Alimentação Escolar. [acessado 2011 nov 22]. Disponível em: http://www.fnde.gov.br/index.php/programasalimentacao-escolar.

7. Sturion GL, Silva MV, Ometto AMH, Furtuoso, Pipitonne MAP. Fatores condicionantes da adesão dos alunos ao Programa de Alimentação Escolar no Brasil. Rev Nutr 2005; 18(2):167-181.

8. Pipitone MAP, Ometto AMH, da Silva MV, Sturion GL, Furtuoso MCO, Oetterer M. Atuação dos conselhos municipais de alimentação escolar na gestão do Programa Nacional de Alimentação Escolar. Rev Nutr 2003; 16(2):143-154.

9. Santos LMP, Santos SMC, Santana LAA, Henrique FCS, Mazza RPD, Santos LAS, Santos LS. Avaliação de políticas públicas de segurança alimentar e combate à fome no período 1995-2002. 4 - Programa Nacional de Alimentação Escolar. Cad Saude Publica 2007; 23(11):2681-2693.

10. Spinelli MAS, Canesqui AM. O programa de alimentação escolar no estado de Mato Grosso: da centralização à descentralização (1979-1995). Rev Nutr 2002; 15(1):105-117.

11. Vianna RPT, Tereso MJA. O Programa de Merenda Escolar de Campinas: análise do alcance e limitacões do abastecimento regional. Rev Nutr 2000; 13(1):41-49.
12. Brasil. Lei ${ }^{\circ} 11.947$ de 16 de junho de 2009. Dispõe sobre o atendimento da alimentação escolar e do Programa Dinheiro Direto na Escola aos alunos da educação básica. Diário Oficial da União 2009; 17 jun.

13. Belik W, Chaim NA. O Programa Nacional de Alimentação Escolar e a gestão municipal: eficiência administrativa, controle social e desenvolvimento local. Rev Nutr 2009; 22(5):595-607.

14. Brasil. FNDE (Fundo Nacional de Desenvolvimento da Educação). Resolução/CD/FNDE no 32, de 10 de agosto de 2006. Estabelece as normas para a execução do Programa Nacional de Alimentação Escolar - PNAE. [acesso 2011 nov 22]. Disponível em: ftp://ftp.fnde.gov.br/web/resolucoes_2006/res 032_10082006.pdf.

15. Brasil. Ministério da Saúde. Conselho Nacional de Saúde. Resolução no. 196 de 10 de outubro de 1996. Diretrizes e Normas Regulamentadoras de Pesquisas Envolvendo Seres Humanos. Diário Oficial da União 1996; out 16.

16. Brasil. Ministério da Saúde. Portaria interministerial no 1.010 de 8 de maio de 2006. Institui as diretrizes para a promoção da alimentação saudável nas escolas de educação infantil, fundamental e nível médio das redes públicas e privadas, em âmbito nacional. Diário Oficial da União 2006; 9 maio.

17. Carvalho AT, Muniz VM, Gomes JF, Samico I. Programa de alimentação escolar no município de João Pessoa - PB, Brasil: as merendeiras em foco. Inter face Comun Saude Educ 2008; 12(27):823-834.

18. World Health Organization (WHO), Pan American Health Organization (OPAS). Escolas Promotoras de Saúde: modelo e guia para a ação. Washington, DC: OPAS; 1996. (HSP/SILOS-36).

19. Chaves LG, Mendes PNR, Brito RR, Botelho RBA. O Programa Nacional de Alimentação Escolar como promotor de hábitos alimentares regionais. Rev Nutr 2009; 22(6):857-866.

20. Cunha E, Sousa AA, Machado NMV. A alimentação orgânica e as ações educativas na escola: diagnóstico para a educação em saúde e nutrição. Cien Saude Colet 2010; 15(1):39-49.

21. Martins PC, Cotta RMM, Mendes FF, Franceschinni SCC, Priore SE, Dias G, Siqueira-Batista R. Conselhos de saúde e a participação social no Brasil: matizes da utopia. Physis 2008; 18(1):105-121.

Artigo apresentado em 02/10/2012

Aprovado em 17/11/2012

Versão final apresentada em 19/12/2012 